\title{
HIGHER-DIMENSIONAL SLICE KNOTS
}

BY D. W. L. SUMNERS ${ }^{1}$

\author{
Communicated by E. Dyer, May 9, 1966
}

The purpose of this paper is to demonstrate the existence of higherdimensional smooth slice (or 0 -concordant) knots of spheres in spheres with generalized Alexander polynomials which are not symmetric and which do not factorize. In particular, this provides a negative answer to questions $B$ and $C$ of Hirsch and Neuwirth [1, Part II]. The method is then extended to provide a generalization of the results of Levine [2].

1. Algebraic theory. Consider an Abelian group $A$ which is finitely generated as a module over the group ring $J Z$ of the infinite cyclic group $Z(t)$ (generated by $t$ ). An $m \times n$ matrix $M=\left(m_{i j}(t)\right)$ whose entries are polynomials in $t$ (integer coefficients) is said to present $A$ as a module if there exists an exact sequence of $J Z$ modules

$$
F_{2} \stackrel{d_{2}}{\rightarrow} F_{1} \rightarrow A \rightarrow 0
$$

where $F_{1}$ and $F_{2}$ are free $J Z$ modules on $\left(x_{1}, \cdots, x_{n}\right)$ and $\left(r_{1}, \cdots, r_{m}\right)$ respectively, and $d_{2}\left(r_{i}\right)=\sum_{j=1}^{n} m_{i j}(t) x_{j}$. See [3].

2. Generalized Alexander polynomials. A smooth $n$-knot is a smooth sphere pair $\left(S^{n+2}, S^{n}\right)$. If $\pi_{1}\left(S^{n+2}-S^{n}\right)=G$, and $G^{\prime}=$ commutator subgroup of $G$, then the universal Abelian covering space $\tilde{X}$ of the knot complement $S^{n+2}-S^{n}$ is the regular covering space corresponding to $G^{\prime}$. That is, $\pi_{1}(\tilde{X})=G^{\prime}$, and the group of covering translations is the Abelian Group $G / G^{\prime}=Z(t)$. The chain groups of $\tilde{X}$, and hence the homology groups $H_{j}(\tilde{X})$ are finitely generated as modules over $J G / G^{\prime}=J Z(t)$, and have presentation matrices $M_{j}$, for all $j$ (See [1]). In the terminology of [3], if $\epsilon_{1(j)}$ is the 1st elementary ideal of $M_{j}$ and $\epsilon_{1(j)}$ is a principal ideal, then define the $j$ th dimensional Alexander polynomial $\Delta_{j}(t)=$ generator of $\epsilon_{1(j)}$. If $M_{j}$ is square, then $\Delta_{j}(t)=\left|M_{j}\right|$, the determinant of $M_{j}$. When $j=1$, the polynomial is the usual Alexander polynomial [4, p. 353]. Also, one can easily define a whole sequence of generalized Alexander polynomials in dimension $j$, each one corresponding to a higher elementary ideal of the presentation matrix $M_{j}$ (See [2].).

${ }^{1}$ Supported by a Marshall Scholarship. 
3. The theorems. In the following, a smooth slice knot is a smooth sphere pair $\left(S^{n+2}, S^{n}\right)$ which bounds a smooth ball pair $\left(B^{n+3}, B^{n+1}\right)$.

Theorem 1. If $n \geqq 2$, and $\mid \leqq j \leqq n / 2$, there exist smooth slice knots $\left(S^{n+2}, S^{n}\right)$ with ith dimensional Alexander Polynomials $\Delta_{i}(t), 1 \leqq i \leqq j$, such that:

(i) $\Delta_{i}(t)=1 \quad 1 \leqq i<j$.

(ii) $\Delta_{j}(t)$ is not symmetric $\left(\Delta_{j}(t) \neq \pm t^{\alpha} \Delta_{j}(1 / t)\right.$, any $\left.\alpha\right)$.

(iii) $\Delta_{j}(t)$ does not factorize (there exists no polynomial $F(t)$ such that $\Delta_{j}(t)= \pm t^{\beta} F(t) F(1 / t)$, some $\left.\beta\right)$.

Proof. The proof is based on the construction in [5] which gives knotted ball pairs in unknotted sphere pairs. The slice knots we are interested in will in all cases be the boundary of a constructed ball pair. In many cases the ball pairs will be precisely those obtained in [5], and the method of construction is exactly the same, but without the worry of embedding the ball pair in an unknotted sphere pair. The method may be described as follows: take an unknotted ball pair $\left(B^{n+3}, B^{n+1}\right)$, and add a solid $j$-handle to $B^{n+3}-B^{n+1}$ by a trivial $S^{j-1}$ embedded in $S^{n+2}-S^{n}=\partial\left(B^{n+3}-B^{n+1}\right)$. Then if $K=\left(B^{n+3}-B^{n+1}\right)$ $\cup h^{j}, K \simeq S^{1} \bigvee S^{j}\left(\simeq\right.$ denotes homotopy equivalence) and $\partial K \simeq S^{1} \bigvee S^{j}$ $\bigvee S^{n+2-j}$. For $j>1$ let $\alpha \in \pi_{j}(\partial K)$ represent the homotopy class of the inclusion map of $S^{j}$ in $S^{1} \bigvee S^{j} \bigvee S^{n+2-j}$, and $t$ represent the action of the generator of $\pi_{1}(\partial K)$ on $\pi_{j}(\partial K)$. When $j=1$, let $\alpha$ represent the path around the handle $h^{1}$ and $\beta$ the path around the $S^{n}$. One adds a handle $h^{j+1}$ whose attaching sphere $S^{j}$ represents the element $2 \alpha-t \alpha$ in $\pi_{j}(\partial K)(j>1)$, or the element $\alpha^{2} \beta \alpha^{-1} \beta^{-1}$ in the case $j=1$. This yields a knotted ball pair with boundary a knotted slice sphere pair.

The proof falls into two cases:

Case 1: $j=1$. The construction gives a smooth slice knot $\left(S^{n+2}, S^{n}\right)$ such that $\pi_{1}\left(S^{n+2}-S^{n}\right)=\left(\alpha, \beta \mid \alpha^{2} \beta \alpha^{-1} \beta^{-1}\right)$. The Fox free derivative process [6] gives us $\Delta_{1}(t)=2-t$, when Abelianization sends $\alpha$ to 1 and $\beta$ to $t$. This means that a result of Fox and Milnor [7] fails to generalize to higher dimensions, and gives us smooth slice knots which are not invertible and not + -amphicheiral (in the sense of [6]). Also, it proves that Neuwirth's group of knot groups [8, Chapter 8], is nontrivial for $n \geqq 2$.

Case 2: $j>1$. The construction can again be used to produce a smooth slice knot $\left(S^{n+2}, S^{n}\right)$ such that $\pi_{1}\left(S^{n+2}-S^{n}\right)=Z(t), \pi_{i}\left(S^{n+2}-S^{n}\right)$ $=0, \quad 1<i<j$, and $\pi_{j}\left(S^{n+2}-S^{n}\right)=\left(t^{i} \alpha \mid 2 \alpha-t \alpha\right)$. This means that $\pi_{1}(\tilde{X})=G^{\prime}=0$, and the Hurewicz Isomorphism Theorem implies $H_{j}(\tilde{X})=\pi_{j}(\tilde{X})=\pi_{j}\left(S^{n+2}-S^{n}\right)$. The matrix presenting $H_{j}(\tilde{X})$ as a 
module over $J G / G^{\prime}=J Z(t)$ is the $1 \times 1$ matrix $(2-t)$, so $\Delta_{j}(t)=2-t$. When $j=n / 2$ ( $n$ even) this answers questions $\mathrm{B}$ and $\mathrm{C}$ of Hirsch and Neuwirth [1] in the negative.

In fact, the construction allows us to prove the following:

Theorem 2. For $n \geqq 2,1 \leqq j \leqq n / 2$, and given any polynomial $F(t)$ such that $F(1)= \pm 1$, then there exists a smooth slice knot $\left(S^{n+2}, S^{n}\right)$ with Alexander polynomials $\Delta_{i}(t), 1 \leqq i \leqq j$, such that $\Delta_{j}(t)=F(t)$.

Proof. The construction and reasoning is exactly the same as in Theorem 1, with the polynomial $F(t)$ becoming a relation in the appropriate homotopy group.

Case 1: $j>1$. Construct the knot as in Theorem 1, but attach $h^{j+1}$ by the element $F(t) \alpha$ instead of $2 \alpha-t \alpha$. This yields a slice knot such that $\pi_{1}\left(S^{n+2}-S^{n}\right)=Z(t), \pi_{i}\left(S^{n+2}-S^{n}\right)=0,1<i<j$, and $\pi_{j}\left(S^{n+2}-S^{n}\right)$ $=\left(t^{i} \alpha \mid F(t) \alpha\right)$. Clearly $\Delta_{j}(t)=F(t)$.

Case $2: j=1$. (I would like to thank Dr. J. F. P. Hudson for pointing out the validity of the theorem in this case.) Given a polynomial $F(t)=\sum_{i=0}^{m} a_{i} t^{i}$, construct the knot as in Theorem 1, adding the second handle by the element $\alpha^{a_{0}} \beta \alpha^{a_{1}} \ldots \beta \alpha^{a_{m}} \beta^{-m}$ instead of $\alpha^{2} \beta \alpha^{-1} \beta^{-1}$. This yields a slice knot such that

$$
\pi_{1}\left(S^{n+2}-S^{n}\right)=\left(\alpha, \beta \mid \alpha^{a_{0}} \beta \alpha^{a_{1}} \cdots \beta \alpha^{a_{m}} \beta^{-m}\right) .
$$

The Fox free derivative process yields $\Delta_{1}(t)=F(t)$, where $\alpha \rightarrow 1$ and $\beta \rightarrow t$ as before. When $n=2$, this provides a very simple proof of the results of Kinoshita [9], who constructed a smooth (not necessarily slice) sphere pair $\left(S^{4}, S^{2}\right)$ corresponding to the given polynomial $F(t)$. One could also compare the results of Terasaka (see [6, p. 136]), who constructed a smooth slice knot $\left(S^{4}, S^{2}\right)$ corresponding to any factorizing polynomial.

Suppose now that we are given a sequence of polynomials $F_{i}(t)$, $1 \leqq i$, such that

(a) for some integer $p, F_{i}(t)$ is a unit (i.e. $\left.F_{i}(t)= \pm t^{\alpha}\right)$ for $i>p$.

(b) $F_{i}(1)= \pm 1$.

(c) $F_{i+1} \mid F_{i}$.

(d) If $\lambda_{i}=F_{i-1} / F_{i}$, then $\lambda_{i+1} \mid \lambda_{i}$.

Then we have the following generalization of the results of Levine [2]:

THEOREM 3. For $n \geqq 2,1 \leqq j \leqq n / 2$, and given any sequence of polynomials $F_{i}$ satisfying (a)-(d), then there exists a smooth slice knot $\left(S^{n+2}, S^{n}\right)$ with the $F_{i}$ as its sequence of generalized Alexander polynomials in dimension $j$. 
Proof. Reasoning as in [2], the problem reduces to finding a slice knot which has a diagonal matrix with entries $\lambda_{i}$ presenting $H_{j}(\tilde{X})$. Theorem 2 tells us that we can find a slice knot for each nonunit $\lambda_{i}$ since $\lambda_{i}(1)= \pm 1$, and we take the connected sum of all these knots to produce the desired one.

\section{REFERENCES}

1. M. W. Hirsch and L. P. Neuwirth, On piecewise-regular n-knots, Ann. of Math. 80 (1964), 594-612.

2. J. Levine, A characterization of knot polynomials, Topology 4 (1965), 135-141.

3. R. H. Crowell, The annihilator of a knot module, Proc. Amer. Math. Soc. 15 (1964), 696-700.

4. - The group $G^{\prime} / G^{\prime \prime}$ of a knot group $G$, Duke Math. J. 30 (1963), 349-354.

5. J. F. P. Hudson and D. W. L. Sumners, Knotted ball pairs in unknotted sphere pairs, J. London Math. Soc. 41 (1966), (to appear.)

6. R. H. Fox, "A quick trip through knot theory," Topology of 3-manifolds and related topics, Prentice-Hall, Englewood Cliffs, New Jersey, 1961, pp. 120-167.

7. R. H. Fox and J. W. Milnor, Singularities of 2-spheres in 4-space and equivalence of knots, Bull, Amer. Math. Soc. 63 (1957), 406.

8. L. P. Neuwirth, Knot groups, Ann. of Math. Studies No. 56, Princeton University, Princeton, N. J., 1965.

9. S. Kinoshita, On the Alexander polynomials of 2-spheres in a 4-sphere, Ann. of Math. 74 (1961), 518-531.

Pembroke College

Cambridge, England 